\title{
Calculations on Lie Algebra of the Group of Affine Symplectomorphisms
}

\author{
Zuhier Altawallbeh \\ Department of Mathematics, Tafila Technical University, Tafila 66110, Jordan \\ Correspondence should be addressed to Zuhier Altawallbeh; zuhier1980@gmail.com
}

Received 13 November 2016; Accepted 4 January 2017; Published 23 January 2017

Academic Editor: Manuel De León

Copyright (C) 2017 Zuhier Altawallbeh. This is an open access article distributed under the Creative Commons Attribution License, which permits unrestricted use, distribution, and reproduction in any medium, provided the original work is properly cited.

\begin{abstract}
We find the image of the affine symplectic Lie algebra $\mathfrak{g}_{n}$ from the Leibniz homology $\mathrm{HL}_{*}\left(\mathfrak{g}_{n}\right)$ to the Lie algebra homology $\mathrm{H}_{*}^{\mathrm{Lie}}\left(\mathfrak{g}_{n}\right)$. The result shows that the image is the exterior algebra $\wedge^{*}\left(w_{n}\right)$ generated by the forms $w_{n}=\sum_{i=1}^{n}\left(\partial / \partial x^{i} \wedge \partial / \partial y^{i}\right)$. Given the relevance of Hochschild homology to string topology and to get more interesting applications, we show that such a map is of potential interest in string topology and homological algebra by taking into account that the Hochschild homology $\operatorname{HH}_{*-1}\left(U\left(\mathfrak{g}_{n}\right)\right)$ is isomorphic to $\mathrm{H}_{*-1}^{\mathrm{Lie}}\left(\mathfrak{g}_{n}, U\left(\mathfrak{g}_{n}\right)^{\text {ad }}\right)$. Explicitly, we use the alternation of multilinear map, in our elements, to do certain calculations.
\end{abstract}

\section{Introduction}

Recall that the group of affine symplectomorphisms, which is the affine symplectic group $\mathrm{AS}_{n}$, is given by all transformations $\Psi: \mathbb{R}^{2 n} \rightarrow \mathbb{R}^{2 n}$ of the form $\Psi(z)=z_{0}+A z$, where $A$ is a $2 n \times 2 n$ symplectic matrix and $z_{0}$ a fixed element of $\mathbb{R}^{2 n}[1]$. The Lie algebra $\mathfrak{g}_{n}$ of AS $p_{n}$ is called the affine symplectic Lie algebra. By using some details and facts from [2,3], Lodder [4] has proved that the structure of the Leibniz homology of $\mathfrak{g}_{n}$ is determined by the exterior algebra of the forms $w_{n}=$ $\sum_{i=1}^{n}\left(\partial / \partial x^{i} \wedge \partial / \partial y^{i}\right)$, as follows: $\operatorname{HL}_{*}\left(\mathfrak{g}_{n}\right) \simeq \wedge^{*}\left(w_{n}\right)$, where $\partial / \partial x^{i}$ and $\partial / \partial y^{i}$ are the unit vector fields parallel to $x_{i}$ and $y_{i}$ axes, respectively, and the Lie algebra homology $\mathrm{H}_{*}^{\mathrm{Lie}}\left(\mathfrak{g}_{n}\right)$ has been proved to have an isomorphic vector space as follows: $\mathrm{H}_{*}^{\mathrm{Lie}}\left(\mathfrak{g}_{n}\right) \simeq \mathrm{H}_{*}\left(s p_{n}, \mathbb{R}\right) \otimes \wedge^{*}\left(w_{n}\right)$, where $\mathrm{H}_{*}\left(s p_{n}, \mathbb{R}\right)$ is the singular homology of the real symplectic Lie algebra $s p_{n}$ and $\wedge^{*}\left(w_{n}\right)=\sum_{k \geq 0} \wedge^{k}\left(w_{n}\right)$.

Here, we find the image of the affine symplectic Lie algebra $\mathfrak{g}_{n}$ from the Leibniz homology $\mathrm{HL}_{*}\left(\mathfrak{g}_{n}\right)$ to the Lie algebra homology $\mathrm{H}_{*}^{\mathrm{Lie}}\left(\mathfrak{g}_{n}\right)$. The result shows that the image is the tensor of a real number with the exterior algebra $\wedge^{*}\left(w_{n}\right)$. We use the alternation of multilinear map, in our elements, to do certain calculations.

Any advances for computations in Hochschild homology are fundamental in string topology because of the high connection between both Hochschild homology and free loop spaces [5]. In this paper, we show that the image of $\mathrm{HL}_{*}\left(\mathfrak{g}_{n}\right)$ in $\mathrm{H}_{*-1}^{\mathrm{Lie}}\left(\mathfrak{g}_{n}\right)$ is important to find the image in Hochschild homology $\mathrm{HH}_{*-1}\left(U\left(\mathfrak{g}_{n}\right)\right)$. In particular, we find the image via the map

$$
\begin{aligned}
j_{*} \circ \pi_{*}: \mathrm{HL}_{*}\left(\mathfrak{g}_{n}\right) & \stackrel{\pi_{*}}{\longrightarrow} \mathrm{H}_{*-1}^{\mathrm{Lie}}\left(\mathfrak{g}_{n}, \mathfrak{g}_{n}\right) \\
& \stackrel{j_{*}}{\longrightarrow} \mathrm{H}_{*-1}^{\mathrm{Lie}}\left(\mathfrak{g}_{n}, U\left(\mathfrak{g}_{n}\right)^{\text {ad }}\right),
\end{aligned}
$$

where the maps $j_{*}$ and $\pi_{*}$ are induced by the chain maps, on the chain level, $\mathrm{CL}_{*}\left(\mathfrak{g}_{n}\right) \stackrel{\pi}{\rightarrow} C_{*-1}^{\mathrm{Lie}}\left(\mathfrak{g}_{n}, \mathfrak{g}_{n}\right)$ and $C_{*-1}^{\text {Lie }}\left(\mathfrak{g}_{n}, \mathfrak{g}_{n}\right) \stackrel{j}{\rightarrow}$ $C_{*-1}^{\text {Lie }}\left(\mathfrak{g}_{n}, U\left(\mathfrak{g}_{n}\right)^{\text {ad }}\right)$, respectively, and $U\left(\mathfrak{g}_{n}\right)^{\text {ad }}$ is the adjoint universal enveloping algebra of $\mathfrak{g}_{n}$. Since $\mathrm{H}_{*-1}^{\mathrm{Lie}}\left(\mathfrak{g}_{n}, U\left(\mathfrak{g}_{n}\right)^{\text {ad }}\right)$ is isomorphic to the Hochschild homology $\mathrm{HH}_{*-1}\left(U\left(\mathfrak{g}_{n}\right)\right)$ [6], we get the image in $\mathrm{HH}_{*-1}\left(U\left(\mathfrak{g}_{n}\right)\right)$.

In symmetric geometry, the study of symplectic algebras is important in manifolds because of the structure of the symplectic group $\mathrm{SP}(2 n, \mathbb{R})$ preserving the transformations of the symplectic vector space at any point of symplectic manifolds, and the reader is kindly requested to refer to [7] to get more applications and interactions with classical mechanics. Moreover, considering position and momentum in the frame of quantum state in physics, symplectic group 
can be considered as an important tool in the phase space. Thus, in the paper, both the source about symplectic algebras and the target related to Hochschild (co)homology make the paper in the intersection field from mathematics to physics.

By referring to [6], we recall that Leibniz homology is a noncommutative theory for Lie algebras, while Hochschild homology is a noncommutative theory for algebras, in the sense that Leibniz homology does not require the skewsymmetry of the bracket for a Lie algebra, while Hochschild homology does not require commutativity of the product in an algebra.

\section{Preliminaries}

For any Lie algebra $\mathfrak{g}$ over a ring $k$, the Lie algebra homology of $\mathfrak{g}$, written $\mathrm{H}_{*}^{\mathrm{Lie}}(\mathfrak{g}, k)$, is the homology of the chain complex $\wedge^{*}(\mathfrak{g})$ which was introduced by Chevalley and Eilenberg in [8]; namely,

$$
k \stackrel{0}{\longleftarrow} \mathfrak{g} \stackrel{[,]}{\longleftarrow} \mathfrak{g}^{\wedge 2} \stackrel{d}{\longleftarrow} \cdots \stackrel{d}{\longleftarrow} \mathfrak{g}^{\wedge(n-1)} \stackrel{d}{\longleftarrow} \mathfrak{g}^{\wedge n} \stackrel{d}{\longleftarrow} \cdots,
$$

where

$$
\begin{aligned}
d\left(g_{1} \wedge g_{2} \wedge \cdots \wedge g_{n}\right)= & \sum_{1 \leq i<j \leq n}(-1)^{i+j}\left[g_{i}, g_{j}\right] \wedge g_{1} \\
& \wedge \cdots \wedge \hat{g}_{i} \wedge \cdots \wedge \hat{g}_{j} \wedge \cdots \\
& \wedge g_{n},
\end{aligned}
$$

where the notation $\hat{\mathfrak{g}}_{i}$ means that element has been deleted. In this paper $\mathrm{H}_{*}^{\mathrm{Lie}}(\mathfrak{g})$ denotes homology with real coefficients, where $k=\mathbb{R}$. Lie homology, with coefficients in the adjoint representation of the universal enveloping algebra $U(\mathfrak{g})^{\text {ad }}$, is the homology of the chain complex $U(\mathfrak{g}) \otimes \wedge^{*}(\mathfrak{g})$ :

$$
\begin{aligned}
U(\mathfrak{g}) & \longleftarrow U(\mathfrak{g}) \otimes \mathfrak{g} \longleftarrow U(\mathfrak{g}) \otimes \mathfrak{g}^{\wedge 2} \longleftarrow \cdots \\
& \longleftarrow U(\mathfrak{g}) \otimes \mathfrak{g}^{\wedge(n-1)} \stackrel{d}{\longleftarrow} U(\mathfrak{g}) \otimes \mathfrak{g}^{\wedge n} \longleftarrow \cdots,
\end{aligned}
$$

where

$$
\begin{aligned}
& d\left(g_{0} \otimes g_{1} \wedge g_{2} \wedge \cdots \wedge g_{n}\right)=\sum_{1 \leq j \leq n}(-1)^{j+1}\left[g_{0}, g_{j}\right] \otimes g_{1} \wedge g_{2} \wedge \cdots \hat{g}_{j} \wedge \cdots \wedge g_{n} \\
& \quad+\sum_{1 \leq i<j \leq n}(-1)^{i+j} g_{0} \otimes\left[g_{i}, g_{j}\right] \wedge g_{1} \wedge \cdots \wedge \hat{g}_{i} \\
& \wedge \cdots \wedge \hat{g}_{j} \wedge \cdots \wedge g_{n} .
\end{aligned}
$$

The canonical projection

$$
\rho: \mathfrak{g} \otimes \wedge^{*}(\mathfrak{g}) \longrightarrow \wedge^{*+1}(\mathfrak{g})
$$

given by $\mathfrak{g} \otimes \mathfrak{g}^{\wedge n} \rightarrow \mathfrak{g}^{\wedge(n+1)}$ is a map of chain complexes and induces a $k$-linear map on homology

$$
\rho_{*}: \mathrm{H}_{n}^{\text {Lie }}(\mathfrak{g}, \mathfrak{g}) \longrightarrow \mathrm{H}_{n+1}^{\mathrm{Lie}}(\mathfrak{g}, k) \text {. }
$$

To see more details, the reader is kindly requested to look at [9].

\section{Leibniz and Hochschild Homology}

Returning to the general setting of any Lie algebra $\mathfrak{g}$ over a ring $k$, we recall that the Leibniz homology [10] of $\mathfrak{g}$, written $\mathrm{HL}_{*}(\mathfrak{g})$, is the homology of the chain complex

$$
k \stackrel{0}{\longleftarrow} \mathfrak{g} \stackrel{[,]}{\longleftarrow} \mathfrak{g}^{\otimes 2} \stackrel{\partial}{\longleftarrow} \cdots \stackrel{\partial}{\longleftarrow} \mathfrak{g}^{\otimes(n-1)} \stackrel{\partial}{\longleftarrow} \mathfrak{g}^{\otimes n} \stackrel{\partial}{\longleftarrow} \cdots,
$$

where

$$
\begin{aligned}
\partial\left(g_{1} \otimes g_{2} \otimes \cdots \otimes g_{n}\right)= & \sum_{1 \leq i<j \leq n}(-1)^{j} g_{1} \otimes g_{2} \otimes \cdots \\
& \otimes g_{i-1} \otimes\left[g_{i}, g_{j}\right] \otimes g_{i+1} \otimes \cdots \\
& \otimes \hat{g}_{j} \otimes \cdots \otimes g_{n} .
\end{aligned}
$$

Definition 1. Let $k$ be a commutative ring and $M$ be a $\mathfrak{g}$ bimodule of an associative (not necessarily commutative) $k$ algebra $\mathfrak{g}$. We define the Hochschild complex $\mathrm{CH}_{*}(\mathfrak{g}, M)$ as the sequence of maps $\mathrm{CH}_{*}(\mathfrak{g}, M): \cdots \stackrel{b}{\rightarrow} M \otimes \mathfrak{g}^{\otimes n} \stackrel{b}{\rightarrow}$ $M \otimes \mathfrak{g}^{\otimes n-1} \stackrel{b}{\rightarrow} \cdots \stackrel{b}{\rightarrow} M \otimes \mathfrak{g} \stackrel{b}{\rightarrow} M$, where the module $M \otimes \mathfrak{g}^{\otimes n}$ is in degree $n$. The Hochschild boundary map $b: M \otimes \mathfrak{g}^{\otimes n} \rightarrow$ $M \otimes \mathfrak{g}^{\otimes n-1}$ is given by

$$
\begin{aligned}
& b\left(m \otimes g_{1} \otimes g_{2} \otimes \cdots g_{n}\right) \\
&:= m g_{1} \otimes g_{2} \otimes \cdots \otimes g_{n}+\sum_{i=1}^{n-1}(-1)^{i} m \otimes g_{1} \otimes \cdots \\
& \otimes g_{i} g_{i+1} \otimes \cdots \otimes g_{n}+(-1)^{n} g_{n} m \otimes g_{1} \otimes \cdots \\
& \otimes g_{n-1}
\end{aligned}
$$

for $m \in M$ and $g_{i} \in \mathfrak{g}$ for all $i=1, \ldots, n$. The homology groups of the Hochschild complex $\mathrm{CH}_{n}(\mathfrak{g}, M)$ are called the Hochschild homology groups $\mathrm{HH}_{n}(\mathfrak{g}, M)$. For $\mathfrak{g}=M$, we write $\mathrm{HH}_{n}(\mathfrak{g})$.

\section{Affine Symplectic Lie Algebra}

We begin by $\left(x_{1}, x_{2}, \ldots, x_{n}, y_{1}, y_{2}, \ldots, y_{n}\right) \in \mathbb{R}^{2 n}$, where $\partial / \partial x^{i}, \partial / \partial y^{i}$ are the unit vector fields parallel to $x_{i}$ and $y_{i}$ axes, respectively. Then the real symplectic Lie algebra $s p_{n}$ has a basis

$$
\begin{aligned}
\mathscr{B}_{1} & =\left\{x_{k} \frac{\partial}{\partial y^{k}}, y_{k} \frac{\partial}{\partial x^{k}} \text { where } k=1,2, \ldots, n, x_{i} \frac{\partial}{\partial y^{j}}\right. \\
& +x_{j} \frac{\partial}{\partial y^{i}}, y_{i} \frac{\partial}{\partial x^{j}}+y_{j} \frac{\partial}{\partial x^{i}} \text { where } 1 \leq i<j \\
& \leq n, y_{j} \frac{\partial}{\partial y^{i}}-x_{i} \frac{\partial}{\partial x^{j}} \text { where } i=1,2, \ldots n, j \\
& =1,2, \ldots, n\} .
\end{aligned}
$$

Let $I_{n}$ be the abelian Lie algebra with the basis $\mathscr{B}_{2}=$ $\left\{\partial / \partial x^{1}, \partial / \partial x^{2}, \ldots, \partial / \partial x^{n}, \partial / \partial y^{1}, \partial / \partial y^{2}, \ldots, \partial / \partial y^{n}\right\}$. The affine 
symplectic Lie algebra $\mathfrak{g}_{n}$ has the basis $\mathscr{B}_{1} \cup \mathscr{B}_{2}$. Thus, there is a short exact sequence of Lie algebras

$$
0 \longrightarrow I_{n} \stackrel{i}{\longrightarrow} \mathfrak{g}_{n} \stackrel{\pi}{\longrightarrow} s p_{n} \longrightarrow 0 \text {. }
$$

In the following example, we find the Lie brackets of the elements in $s p_{2}$ by taking into account the basic elements illustrated above.

Example 2. The basis $\mathscr{B}_{1}$ of the real symplectic Lie algebra $s p_{2}$ contains exactly these elements

$$
\begin{gathered}
x_{1} \frac{\partial}{\partial y^{1}}, \\
x_{2} \frac{\partial}{\partial y^{2}}, \\
y_{1} \frac{\partial}{\partial x^{1}}, \\
y_{2} \frac{\partial}{\partial x^{2}}, \\
x_{1} \frac{\partial}{\partial y^{2}}+x_{2} \frac{\partial}{\partial y^{1}}, \\
y_{1} \frac{\partial}{\partial x^{2}}+y_{2} \frac{\partial}{\partial x^{1}}, \\
y_{1} \frac{\partial}{\partial y^{1}}-x_{1} \frac{\partial}{\partial x^{1}}, \\
y_{2} \frac{\partial}{\partial y^{1}}-x_{1} \frac{\partial}{\partial x^{2}}, \\
y_{1} \frac{\partial}{\partial y^{2}}-x_{2} \frac{\partial}{\partial x^{1}}, \\
\frac{\partial}{\partial y^{2}}-x_{2} \frac{\partial}{\partial x^{2}},
\end{gathered}
$$

which can be denoted by $e_{1}, e_{2}, \ldots, e_{10}$, respectively. It is known that $\left[e_{i}, e_{i}\right]=0$ and $\left[e_{i}, e_{j}\right]=-\left[e_{j}, e_{i}\right]$ for all $i, j=$ $1,2, \ldots, n$. By taking the Lie brackets of the others, it follows that

$$
\begin{aligned}
& {\left[e_{1}, e_{2}\right]=0,} \\
& {\left[e_{1}, e_{3}\right]=x_{1} \frac{\partial}{\partial x^{1}}-y_{1} \frac{\partial}{\partial y^{1}}=-e_{7},}
\end{aligned}
$$

$$
\begin{aligned}
& {\left[e_{1}, e_{4}\right]=0,} \\
& {\left[e_{1}, e_{5}\right]=0,} \\
& {\left[e_{1}, e_{6}\right]=x_{1} \frac{\partial}{\partial x^{2}}-y_{2} \frac{\partial}{\partial y^{1}}=-e_{8} .}
\end{aligned}
$$

Now we take $\left[e_{1}, e_{7}\right]=2 x_{1}\left(\partial / \partial y^{1}\right)=2 e_{1}$, which means that $e_{1}$ is the Eigenvector of $\left[e_{1}, e_{7}\right]$. Similarly, if we continue the computations, we get that $e_{i}$ is the Eigenvector not only for the bracket $\left[e_{7}, e_{i}\right]$ but also for $\left[e_{10}, e_{i}\right]$ for all $i=1, \ldots n$.

The above example shows that the Cartan subalgebra of $s p_{2}$ is $\left\{e_{7}, e_{10}\right\}$ which is the tangent of the maximal torus subset in the Lie group $\mathrm{SP}(2, \mathbb{R})$.

\section{The Image of $\mathrm{HL}_{*}\left(\mathfrak{g}_{n}\right)$ in $\mathrm{H}_{*}^{\mathrm{Lie}}\left(\mathfrak{g}_{n}\right)$}

By convention, we denote the affine symplectic Lie algebra by $\mathfrak{g}_{n}$. There is a canonical projection $T\left(\mathfrak{g}_{n}\right) \rightarrow \wedge^{*}\left(\mathfrak{g}_{n}\right)$, where $T\left(\mathfrak{g}_{n}\right)$ is the tensor algebra of $\mathfrak{g}_{n}$ and $\wedge^{*}\left(\mathfrak{g}_{n}\right)$ is the exterior algebra of $\mathfrak{g}_{n}$, which is naturally defined by $\pi^{\prime}: \mathfrak{g}_{n}^{\otimes l} \rightarrow \mathfrak{g}_{n}^{\wedge l}$ for $l \geq 0$ Thus, the map $\pi^{\prime}$ induces a $k$-linear map on homology

$$
\pi_{*}^{\prime}: \mathrm{HL}_{*}\left(\mathfrak{g}_{n}\right) \rightarrow \mathrm{H}_{*}^{\mathrm{Lie}}\left(\mathfrak{g}_{n}\right) .
$$

From [4], there are these two vector spaces isomorphisms $\mathrm{HL}_{*}\left(\mathfrak{g}_{n}\right) \simeq \wedge^{*}\left(w_{n}\right)$ and $\mathrm{H}_{*}^{\mathrm{Lie}}\left(\mathfrak{g}_{n}\right) \simeq \mathrm{H}_{*}\left(s p_{n}, \mathbb{R}\right) \otimes \wedge^{*}\left(w_{n}\right)$. Let us start with the element $w_{n}=\sum_{i=1}^{n}\left(\partial / \partial x^{i} \wedge \partial / \partial y^{i}\right) \in \operatorname{HL}_{*}\left(\mathfrak{g}_{n}\right)$.

By using the alternation multilinear form, we can rewrite the elements from the wedge notation into tensor product by taking into account the signs of the permutations, so

$$
\begin{aligned}
\pi_{*}^{\prime}\left(w_{n}\right) & =\pi_{*}^{\prime}\left(\sum\left(\frac{\partial}{\partial x^{i}} \wedge \frac{\partial}{\partial y^{i}}\right)\right) \\
& =\pi_{*}^{\prime}\left(\frac{1}{2} \sum_{i=1}^{n}\left(\frac{\partial}{\partial x^{i}} \otimes \frac{\partial}{\partial y^{i}}-\frac{\partial}{\partial y^{i}} \otimes \frac{\partial}{\partial x^{i}}\right)\right) \\
& =\frac{1}{2} \sum_{i=1}^{n}\left(\frac{\partial}{\partial x^{i}} \wedge \frac{\partial}{\partial y^{i}}-\frac{\partial}{\partial y^{i}} \wedge \frac{\partial}{\partial x^{i}}\right) \\
& =\frac{1}{2} \sum_{i=1}^{n}\left(2 \frac{\partial}{\partial x^{i}} \wedge \frac{\partial}{\partial y^{i}}\right)=w_{n} .
\end{aligned}
$$

For more general setting, let us take $\wedge^{2} w_{n} \in \mathrm{HL}_{*}\left(\mathfrak{g}_{n}\right)$, so we get

$$
\begin{aligned}
\pi_{*}^{\prime}\left(\wedge^{2} w_{n}\right) & =\pi_{*}^{\prime}\left(\sum_{i=1}^{n}\left(\frac{\partial}{\partial x^{i}} \wedge \frac{\partial}{\partial y^{i}}\right) \wedge \sum_{i=1}^{n}\left(\frac{\partial}{\partial x^{i}} \wedge \frac{\partial}{\partial y^{i}}\right)\right) \\
& =\pi_{*}^{\prime}\left(\left(\frac{\partial}{\partial x^{1}} \wedge \frac{\partial}{\partial y^{1}}\right) \wedge \sum_{i=1}^{n}\left(\frac{\partial}{\partial x^{i}} \wedge \frac{\partial}{\partial y^{i}}\right)+\cdots+\left(\frac{\partial}{\partial x^{n}} \wedge \frac{\partial}{\partial y^{n}}\right) \wedge \sum_{i=1}^{n}\left(\frac{\partial}{\partial x^{i}} \wedge \frac{\partial}{\partial y^{i}}\right)\right) \\
& =\pi_{*}^{\prime}\left(2 \sum_{1 \leq i<j \leq n}\left(\frac{\partial}{\partial x^{i}} \wedge \frac{\partial}{\partial y^{i}} \wedge \frac{\partial}{\partial x^{j}} \wedge \frac{\partial}{\partial y^{j}}\right)\right)
\end{aligned}
$$




$$
\begin{aligned}
& =\pi_{*}^{\prime}\left(2\left(\frac{1}{24} \sum_{\substack{1 \leq i<j \leq n \\
\sigma \in S_{4}}}\left(\operatorname{sgn}(\sigma)\left[\left(\frac{\partial}{\partial x^{i}}\right)_{\sigma(1)} \otimes\left(\frac{\partial}{\partial y^{i}}\right)_{\sigma(2)} \otimes\left(\frac{\partial}{\partial x^{j}}\right)_{\sigma(3)} \otimes\left(\frac{\partial}{\partial y^{j}}\right)_{\sigma(4)}\right]\right)\right)\right) \\
& =2 \sum_{1 \leq i<j \leq n}\left(\frac{\partial}{\partial x^{i}} \wedge \frac{\partial}{\partial y^{i}} \wedge \frac{\partial}{\partial x^{j}} \wedge \frac{\partial}{\partial y^{j}}\right)=\wedge^{2} w_{n} .
\end{aligned}
$$

Thus

$$
\pi_{*}^{\prime}\left(\wedge^{2} w_{n}\right)=\wedge^{2} w_{n} \in \mathrm{H}_{*}^{\mathrm{Lie}}\left(\mathfrak{g}_{n}\right)
$$

The result makes sense because $\mathrm{H}_{*}^{\mathrm{Lie}}\left(\mathfrak{g}_{n}\right) \simeq \mathrm{H}_{*}\left(s p_{n}, \mathbb{R}\right) \otimes$ $\wedge^{*}\left(w_{n}\right)$ and $\mathrm{H}_{0}\left(s p_{n}, \mathbb{R}\right) \otimes \wedge^{*}\left(w_{n}\right)=\mathbb{R} \otimes \wedge^{*}\left(w_{n}\right) \simeq \wedge^{*}\left(w_{n}\right)$.

\section{The Image in the Hochschild Homology}

Hochschild homology plays a significant role in string topology, so any progress on computations about this kind of homology will be interesting for mathematicians and for those who are working in theoretical physics. First, we find the nonzero images of Leibniz homology $\mathrm{HL}_{*}\left(\mathfrak{g}_{n}\right)$ in the Lie algebra homology $\mathrm{H}_{*-1}^{\mathrm{Lie}}\left(\mathfrak{g}_{n}, U\left(\mathfrak{g}_{n}\right)^{\text {ad }}\right)$ of the adjoint universal enveloping algebra $U\left(\mathfrak{g}_{n}\right)^{\text {ad }}$. In particular, we find the image via the map

$$
\begin{aligned}
j_{*} \circ \pi_{*}: \mathrm{HL}_{*}\left(\mathfrak{g}_{n}\right) \stackrel{\pi_{*}}{\longrightarrow} \mathrm{H}_{*-1}^{\mathrm{Lie}}\left(\mathfrak{g}_{n}, \mathfrak{g}_{n}\right) \\
\stackrel{j_{*}}{\longrightarrow} \mathrm{H}_{*-1}^{\mathrm{Lie}}\left(\mathfrak{g}_{n}, U\left(\mathfrak{g}_{n}\right)^{\mathrm{ad}}\right),
\end{aligned}
$$

where the maps $j_{*}$ and $\pi_{*}$ are induced by the chain maps $\pi$ and $j$ on the chain level. Naturally $\pi$ and $j$ can be defined as follows: $\pi: \mathfrak{g}_{n}^{\otimes l} \rightarrow \mathfrak{g}_{n} \otimes \mathfrak{g}_{n}^{\wedge(l-1)}$ and the inclusion $j: \mathfrak{g}_{n} \otimes$ $\mathfrak{g}_{n}^{\wedge(l-1)} \hookrightarrow U\left(\mathfrak{g}_{n}\right) \otimes \mathfrak{g}_{n}^{\wedge(l-1)}$. It is not difficult to prove that $\pi$ and $j$ are chain maps. Now if we are trying to find $\pi_{*}\left(w_{n}\right)$, where $w_{n} \in \mathrm{HL}_{*}\left(\mathfrak{g}_{n}\right)$, we get similar procedure steps as we have done above, by taking into account that $\pi$ is different a little bit from the map $\pi^{\prime}$ and we will get the same result. I mean $\pi_{*}\left(\wedge^{l} w_{n}\right)=$ $\wedge^{l} w_{n}$. The image result $\wedge^{l} w_{n}$ is in $\mathrm{H}_{*-1}^{\mathrm{Lie}}\left(\mathfrak{g}_{n}, \mathfrak{g}_{n}\right)$ because in [4] it was proven that $\mathrm{H}_{*-1}^{\mathrm{Lie}}\left(\mathfrak{g}_{n}, \mathfrak{g}_{n}\right) \simeq \mathrm{H}_{*}\left(s p_{n}, \mathbb{R}\right) \otimes \overline{\Lambda^{*}}\left(w_{n}\right)$, where $\overline{\wedge^{*}}\left(w_{n}\right)=\sum_{l \geq 1} \wedge^{l} w_{n}$. After taking the induced map $j_{*}$, we get $j_{*}\left(\pi_{*}\left(\wedge^{l} w_{n}\right)\right)=\wedge^{l} w_{n}$ in $\mathrm{H}_{*-1}^{\mathrm{Lie}}\left(\mathfrak{g}_{n}, U\left(\mathfrak{g}_{n}\right)^{\text {ad }}\right)$. If we put the mentioned homological algebras in more general setting as operadic theory and generalize the above result in category theory, it will be more and more applicable in many different fields of study. To see how the homological algebra meets operad, we can read [11].

Definition 3 (the antisymmetrization map $\epsilon_{n}$ ). Suppose that $\mathfrak{g}$ and $M$ as they were in the previous definition. We define [6] the antisymmetrization map $\epsilon_{n}: M \otimes \wedge^{n} \mathfrak{g} \rightarrow M \otimes \mathfrak{g}^{\otimes n}$ as one that sends the element $m \otimes g_{1} \wedge \cdots \wedge g_{n}$ (for $m \in M$ and $g_{i} \in \mathfrak{g}$ for all $\left.i=1, \ldots, n\right)$ to $\epsilon_{n}\left(m \otimes g_{1} \otimes \cdots \otimes g_{n}\right)$ by $\epsilon_{n}:=\sum_{\sigma \in S_{n}} \operatorname{sgn}(\sigma) \sigma$, where $\sigma$ is a permutation in the symmetric group $S_{n}$ on the set of indices $\{1, \ldots, n\}$, and $\sigma$ acts on (the left of) $m \otimes g_{1} \otimes \cdots \otimes g_{n}$ by $\sigma \cdot\left(m \otimes g_{1} \otimes g_{2} \otimes \cdots g_{n}\right)=$ $m \otimes g_{\sigma^{-1}(1)} \otimes \cdots \otimes g_{\sigma^{-1}(n)}$.

Theorem 4. From page 98 of [6], we know that if $\mathfrak{g}$ is a Lie Kalgebra and $M$ is a $U(\mathfrak{g})$-bimodule, then we have the following isomorphism: $H_{*}(U(\mathfrak{g}), M) \stackrel{\epsilon_{*}}{\simeq} H_{*}^{L i e}\left(\mathfrak{g}, M^{a d}\right)$. For $M=U(\mathfrak{g})$, we get $H H_{*}(U(\mathfrak{g})) \stackrel{\epsilon_{*}}{\sim} H_{*}^{L i e}\left(\mathfrak{g}, U(\mathfrak{g})^{a d}\right)$.

By applying the above theorem, we get the image of $\mathrm{HL}_{*}\left(\mathfrak{g}_{n}\right)$ in the Hochschild homology $\mathrm{HH}_{*-1}\left(U\left(\mathfrak{g}_{n}\right)\right)$ as $\left(\epsilon_{*}\right.$ 。 $\left.j_{*} \circ \pi_{*}\right)\left(w_{n}\right)=w_{n}$ for any $w_{n} \in \operatorname{HL}_{*}\left(\mathfrak{g}_{n}\right)$.

Corollary 5. For the affine symplectic Lie algebra $\mathfrak{g}_{n}$, the image of $H_{*}\left(\mathfrak{g}_{n}\right)$ in the Hochschild homology $H_{*-1}\left(U\left(\mathfrak{g}_{n}\right)\right)$ can be identified injectively as the exterior algebra $\wedge^{*}\left(w_{n}\right)$.

\section{Relation to String Topology and Hochschild Cohomology}

Recall that string topology is the study of the algebraic and differential topology of the spaces of paths and loops in compact and oriented manifolds. In this paper, consider a symplectic manifold $M$, so $M$ is canonically oriented by its symplectic forms and it is closed manifold because the forms are closed. Actually, the operations of the loop homology algebra of a manifold are very difficult to compute, but there are several conjectures connecting the string topology with algebraic structures on the Hochschild cohomology of algebras related to the manifold. Thus it is worthy to find the nonzero image in the Hochschild cohomology $\mathrm{HH}^{*-1}\left(U\left(\mathfrak{g}_{n}\right)\right)$ of the associative algebra $U\left(\mathfrak{g}_{n}\right)$.

Although it is not that easy to compute Hochschild cohomology in general, still there are some ways to do it. In this paper, we know from the previous section that the elements in $\mathrm{HL}_{*}\left(\mathfrak{g}_{n}\right)$ are mapped injectively to $\mathrm{HH}_{*-1}\left(U\left(\mathfrak{g}_{n}\right)\right)$. In other words, $\mathrm{HH}_{*-1}\left(U\left(\mathfrak{g}_{n}\right)\right)$ contains $\wedge^{*}\left(w_{n}\right)$ as a direct summand. Now, we know that

$$
\mathrm{HH}^{*-1}\left(U\left(\mathfrak{g}_{n}\right)\right) \simeq \operatorname{Hom}\left(\mathrm{HH}_{*-1}\left(U\left(\mathfrak{g}_{n}\right)\right), \mathbb{R}\right)
$$

Taking into account that $\wedge^{*}\left(w_{n}^{*}\right)$ is the dual space of $\wedge^{*}\left(w_{n}\right)$, where $w_{n}^{*}=d x^{i} \wedge d y^{i}$ and $d x^{i}$ is dual of $\partial / \partial x^{i}$ and $d y^{i}$ is dual of $\partial / \partial y^{j}$, we end up with this following result about the image in Hochschild cohomology of the given algebra.

Corollary 6. The Hochschild cohomology $H H^{*-1}\left(U\left(\mathfrak{g}_{n}\right)\right)$ contains $\wedge^{*}\left(w_{n}^{*}\right)$ as a direct summand. 
As an algebraic point of departure and theoretical physics point of view, the Hochschild cohomology $\mathrm{HH}^{*}(A)$ of an associative algebra $A$ has natural product with a Lie type bracket of degree -1 , satisfying Jacobi identity and graded anticommutativity such that both natural product and Lie type bracket are compatible to make $\operatorname{HH}^{*}(A)$ a Gerstenhaber algebra. Furthermore, the Gerstenhaber algebra structure can be viewed as algebraic properties of the loop homology algebra of a manifold. Here, we concentrate our work by setting $A=U\left(\mathfrak{g}_{n}\right)$.

\section{Competing Interests}

The author declares no competing interests.

\section{References}

[1] D. McDuff and D. Salamon, Introduction to Symplectic Topology, Oxford University Press, 2nd edition, 1998.

[2] P. J. Hilton and U. Stammbach, A Course in Homological Algebra, Springer, Berlin, Germany, 1971.

[3] G. Hochschild and J.-P. Serre, "Cohomology of Lie algebras," Annals of Mathematics, vol. 57, pp. 591-603, 1953.

[4] J. M. Lodder, "Lie algebras of Hamiltonian vector fields and symplectic manifolds," Journal of Lie Theory, vol. 18, no. 4, pp. 897-914, 2008.

[5] R. L. Cohen, K. Hess, and A. A. Voronov, String Topology and Cyclic Homology, Advanced Courses in Mathematics-CRM Barcelona, 2006.

[6] J.-L. Loday, Cyclic Homology, vol. 301 of Fundamental Principles of Mathematical Sciences, Springer, Berlin, Germany, 2nd edition, 1998.

[7] V. I. Arnold, Mathematical Methods of Classical Mechanics, Graduate Texts in Mathematics, vol. 60, Springer, Berlin, Germany, 1989.

[8] C. Chevalley and S. Eilenberg, "Cohomology theory of Lie groups and Lie algebras," Transactions of the American Mathematical Society, vol. 63, pp. 85-124, 1948.

[9] J. M. Lodder, "From Leibniz homology to cyclic homology," KTheory, vol. 27, no. 4, pp. 359-370, 2002.

[10] T. Pirashvili, "On Leibniz homology," Annales de l'Institut Fourier, vol. 44, no. 2, pp. 401-411, 1994.

[11] J.-L. Loday and B. Vallette, Algebraic Operads, vol. 346 of Fundamental Principles of Mathematical Sciences, Springer, Heidelberg, Germany, 2012. 


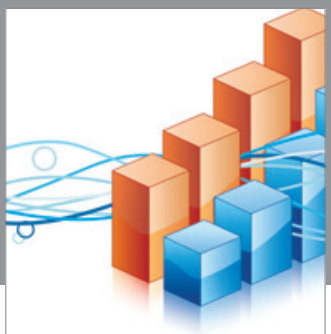

Advances in

Operations Research

vatem alat4

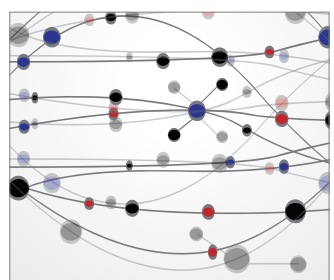

\section{The Scientific} World Journal
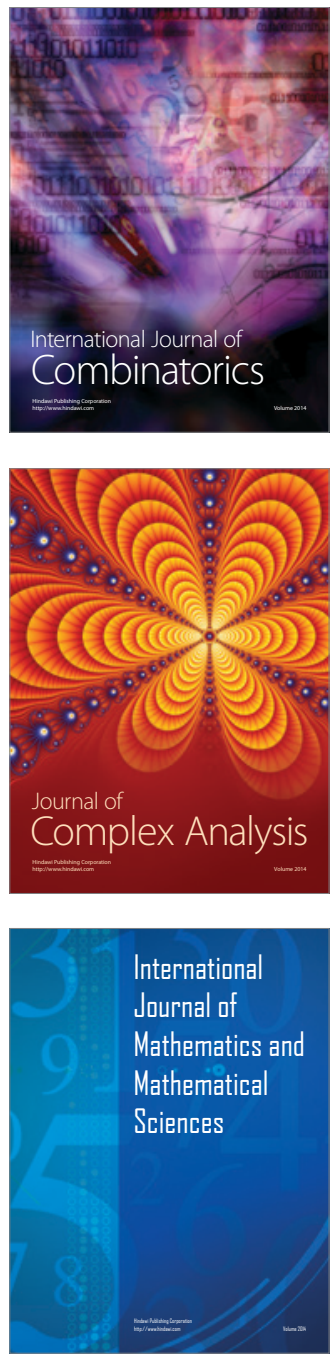
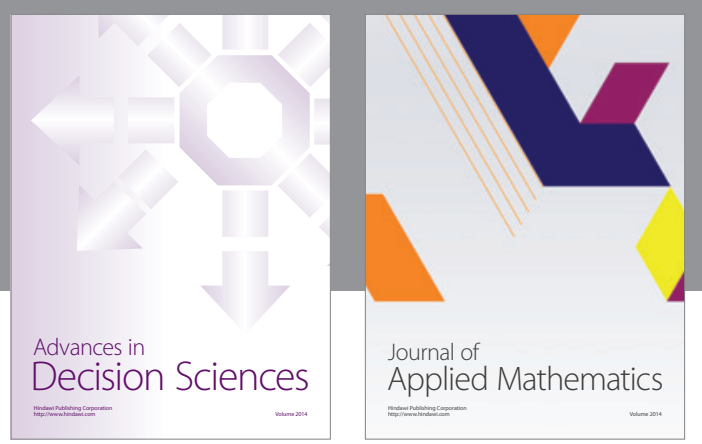

Algebra

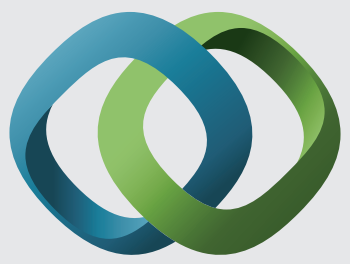

\section{Hindawi}

Submit your manuscripts at

https://www.hindawi.com
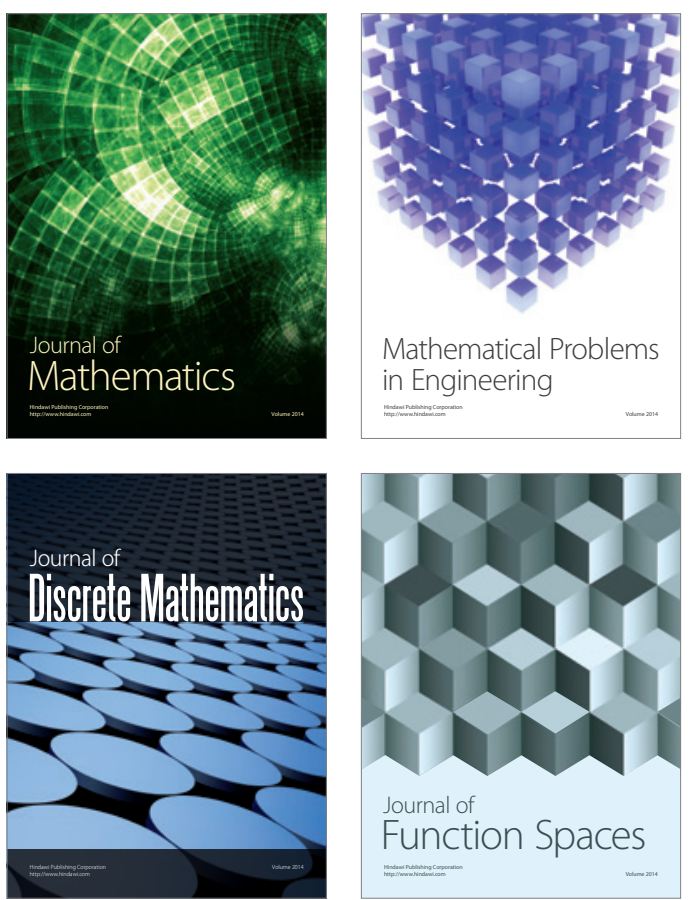

Mathematical Problems in Engineering
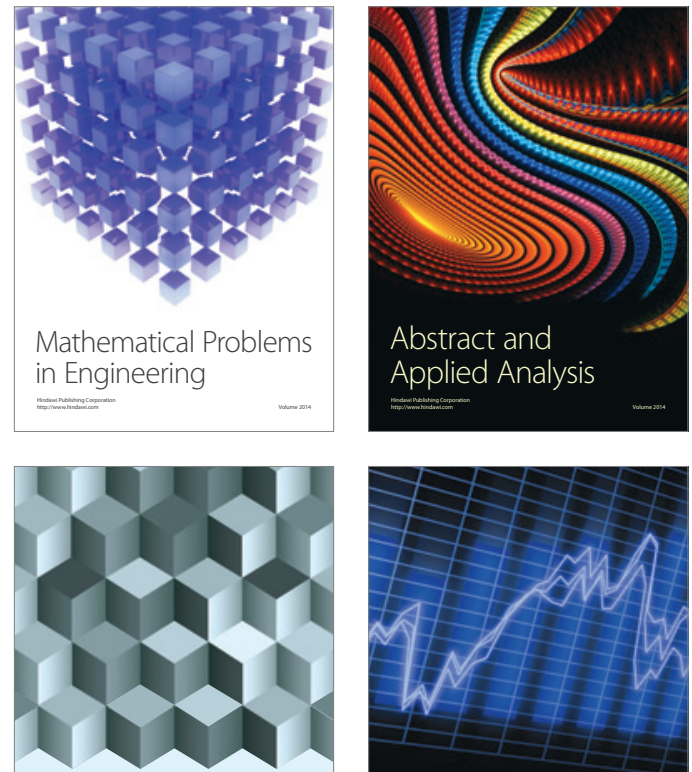

Journal of

Function Spaces

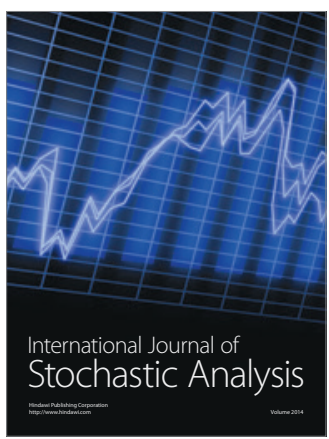

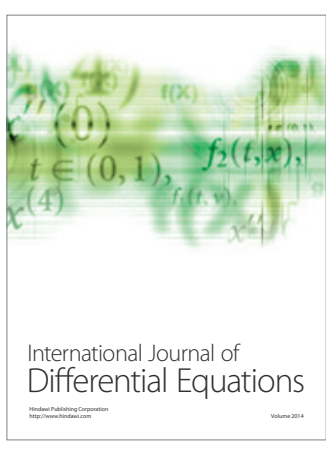
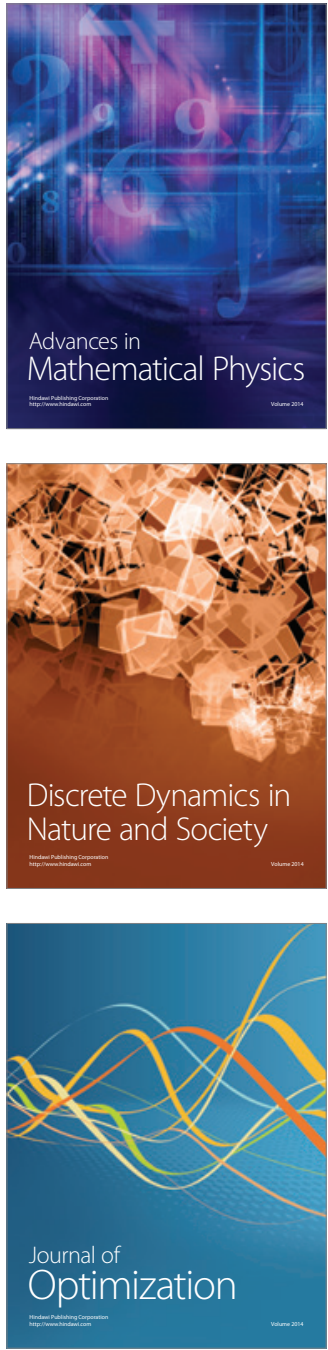